\title{
Endoscopic endodontic microsurgery: 2-year evaluation of healing and functionality
}

\author{
Silvio Taschieri(a) \\ Massimo Del Fabbro(b) \\ (a) MD, DDS, Head of the Endodontics \\ Section; (b)BSc, PhD, Head of the Oral \\ Physiology Section - IRCCS Istituto \\ Ortopedico Galeazzi, Department of Health \\ Technologies, University of Milan, Milan, \\ Italy.
}

\begin{abstract}
This prospective clinical study aimed to evaluate the benefits of the endoscope as an aid to root-end management, and to assess the treatment outcome during 2 years following surgery. Forty-three endodontic surgical procedures in 30 patients were performed with the aid of an endoscope and followed for a period of 2 years. Radiographic criteria and clinical evaluation were used to assess the outcome. All cases were evaluated in terms of healing and functionality. $91.1 \%$ and $90.7 \%$ of the teeth evaluated after 1 and 2 years, respectively, were classified as successful. We found no statistically significant differences for both healing and functionality between the 1- and 2-year evaluations. No difference related to tooth type or tooth location was found at the 2-year follow-up. Fisher's exact test was used to statistically assess the difference between successful and unsuccessful cases for each of the variables considered. The endoscope can be an aid for endodontic surgical procedures in terms of both periapical healing and functionality up to 2 years follow-up.
\end{abstract}

Descriptors: Apicoectomy; Endoscopy; Oral surgical procedures.

Corresponding author:

Massimo Del Fabbro

IRCCS Istituto Ortopedico Galeazzi

Via R. Galeazzi 4

20161 - Milano - Italy

E-mail: massimo.delfabbro@unimi.it

Received for publication on Oct 29, 2007

Accepted for publication on Jun 04, 2008 


\section{Introduction}

Microsurgical instruments and ultrasonic retrotips significantly improved the outcome of periradicular surgery when compared with traditional techniques. ${ }^{1}$ These devices brought advantages for root-end management. ${ }^{1,2}$ At the same time, the use of well-focused illumination and magnification devices has been recommended as a standard of care in periradicular surgery. ${ }^{3}$ Rubinstein, Kim $^{4,5}$ (1999, 2002) have reported very high success rates after periradicular surgery, advocating that the use of a surgical microscope might have been a decisive factor contributing to such an excellent outcome. Recently, there has been a growing interest in endoscopy for optimizing visualization. ${ }^{6-11}$

To date, its use in endodontic surgery has been shown mostly in a few technical reports. $6,7,9,10,12$ These articles reported advantages using the endoscope such as: it is a versatile and expandable system, easily transportable, and has excellent illumination and good magnification; no mirrors are required; the learning curve is short. Due to its non fixed field of vision, the endoscope allows viewing of a treatment field at various angles and distances without losing depth of field and focus. In using the microscope, when increasing the magnitude, any movement of either the microscope or the patient will cause the surgical field to become out of focus. This is one drawback of the microscope when used as an aid to enhance visualization of the surgical field. In the authors' experience using both magnification devices, though recognizing the microscope's usefulness, they have observed that the depth-of-field perception that an operator has using an endoscope is quite similar to the perception that a surgeon has with the naked eye. ${ }^{12}$

Another advantage in the use of an endoscope is that its control is more tactile than that of a microscope. Regulating the endoscope for focusing and zooming using just one finger is faster and more comfortable, and the surgeon can hold the endoscope and the camera in one hand with the pen grasp, whereas, with the microscope, it is sometimes necessary to stop and manually adjust the focus and zoom using one or both hands. The operator can examine the morphological aspects of the roots from almost any angulation in a very short time. This takes longer using a microscope and sometimes it is impossible, especially in the posterior jaws. It is also possible to see behind the roots and ascertain the presence of periradicular lesions and, if necessary, remove them. This is much more complicated when the operator has to use a microscope and the retro-mirror.

The operator can also ascertain the presence of a through-and-through lesion and visualize the size of the bone lesion in the palatal or lingual plate. Using a microscope it is not always possible to achieve the same accurate results.

The rod-lens system of the endoscope allows a good visualization even in the presence of irrigation fluids, so the operator can use retro-tips with sterile water irrigation to avoid overheating and at the same time have lens cleaning. In contrast, using the microscope and the retro-mirror the operator has often to stop in order to clean the mirror. During adjustment of the viewing angle, the endoscope lens position can be misplaced. Once the correct position is lost, however, it is easier to find it again as compared to the microscope.

With regard to the clinical application of endoscopy in periradicular surgery, a few short-term prospective studies have been published recently. ${ }^{8,9}$ In both studies, the authors reported a better outcome in the group using the endoscope as compared with the control group.

The main objectives of this prospective clinical study were to evaluate the clinical advantages of using an endoscope during root-end management and to compare the outcome of periradicular surgery in terms of healing and functionality at 1- and 2 -years follow-up.

\section{Material and Methods}

All patients requiring surgical treatment were recruited during a period of 34 months (from December 2001 to October 2004) in a university clinic and in a private practice setting. Most of the patients enrolled in the present study were also included in one arm of a previously reported comparative prospective study that assessed 1-year outcomes only. ${ }^{8}$ The study was conducted according to the ethic standards of the Helsinki Declaration (1975), reviewed 
in 1983.

\section{Patient selection and inclusion criteria}

The following criteria were adopted for case selection:

1. The tooth treated surgically showed a periradicular lesion of strictly endodontic origin and the non-surgical retreatment was considered unfeasible or had previously failed.

2. The tooth treated surgically exhibited an adequate final restoration with no clinical evidence of coronal leakage.

3. The apical root canal had $6 \mathrm{~mm}$ or more without the presence of a post.

4. Periradicular surgery was carried out only in the absence of acute symptoms.

5. The patient did not have general medical contraindications for oral surgical procedures (ASA-1 or ASA-2).

The following exclusion criteria were applied:

1. Teeth with pathoses associated with vertical root fracture.

2. Teeth with perforation of the furcation area or lateral canal walls.

3. Teeth with traumatic injuries.

4. Severe periodontal bone loss detected with a periodontal probe (5 $\mathrm{mm}$ probing depth).

All teeth with periapical lesions diagnosed radiographically were treated with periradicular surgery. Each patient was given written information about the surgical procedure and the necessary follow-up care; they were also given the opportunity to withdraw from the study. A consent form was signed if they agreed.

\section{Surgical procedure and management of the resected root end}

Full mucoperiosteal tissue flap designs were used. The type of flap design (rectangular, marginal or submarginal) varied according to the periodontal status. The endodontic surgical flap was reflected and carefully retracted. The undermining elevation flap reflection technique was used. Surgical access to the root was then made through the cortical bone using a round bur. Shaving of the bone was performed with a brush-stroke approach, using light pressure, low rotation speed, and avoiding prolonged contact of the bur with the osseous surface. This was done under constant copious sterile water irrigation. The periradicular lesion was removed with sharp bone curettes and angled periodontal curettes. The curetted tissue was placed in $10 \%$ formalin solution for pathologic diagnosis. After exposure of the root end, a straight fissure bur in a hand-piece was positioned perpendicular to the long axis of the root and then, beginning from the apex, cutting coronally, 2.5 to $3 \mathrm{~mm}$ of the root end was shaved away. The bur was moved from mesial to distal at the desired angle (little or no bevel), shaving the root-end surface smooth and flat. All of these procedures were performed using magnification loupes $(4.3 \mathrm{X})$ with a headlight. After rootend resection, surgical procedures were performed with an endoscope. In this study, the root ends were examined using a 6 - $\mathrm{cm}$-long and 3 -mm-wide endoscope with a viewing angle of $70^{\circ}$ (Hopkins Tele-Otoscope $70^{\circ}$, Karl Storz GmbH, Tuttlingen, Germany) (Figure 1). Prior to root-end preparation, local haemostasis was achieved using bone wax. Root-end cavities were prepared using zirconium nitrate retro-tips (Figures 2-3) (Dentsply; Maillefer Instruments, Ballaigues, Switzerland) driven by an ultrasonic device unit (Piezon master 400; EMS, Nyon, Switzerland). All root-end cavities were done setting the ultrasonic device unit at no more than

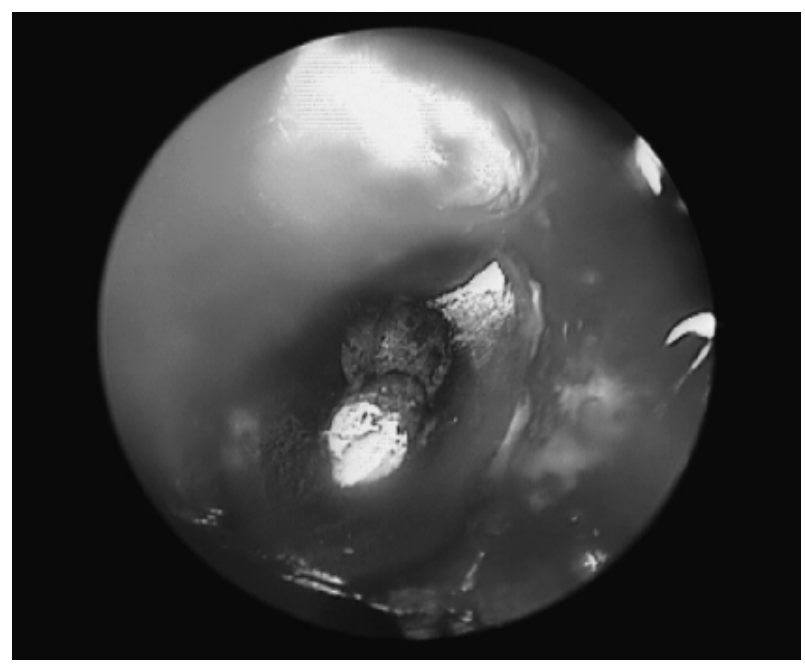

Figure 1 - Endoscopic view of the surface of the resected root end. 


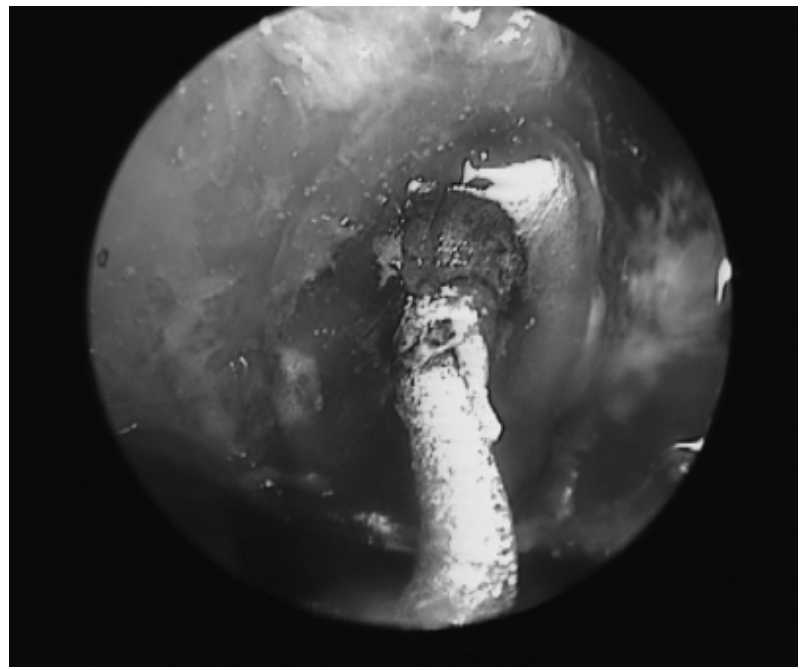

Figure 2 - Endoscopic view of the retro-tip positioned in the orifice of the root canal system.

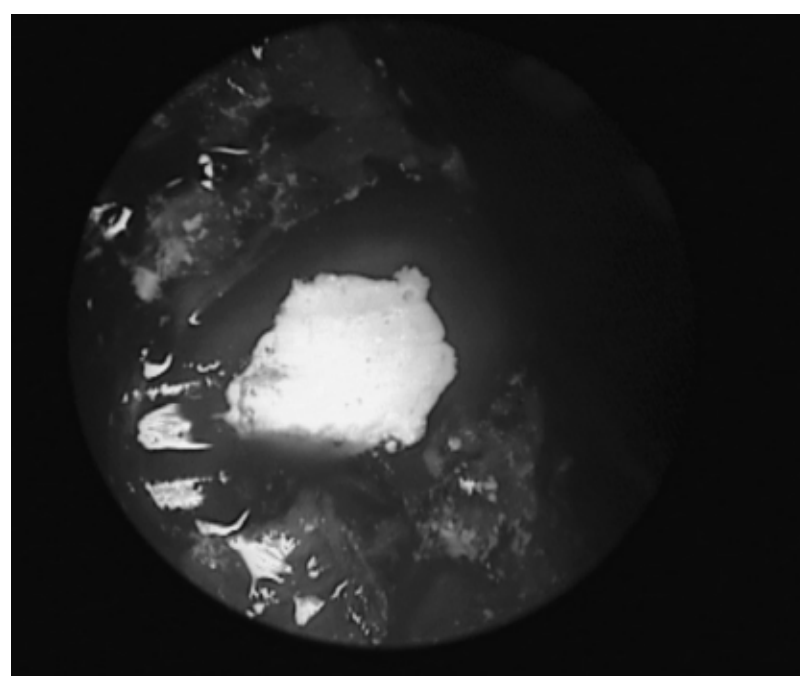

Figure 4 - Surface of the resected root end after root-end filling.

half power, under constant copious sterile water irrigation to avoid overheating. The retro-tips had a 3-mm-long tip that allowed a well-defined parallel preparation of 2.5- to $3-\mathrm{mm}$ in depth. The root-end cavities were then dried using a paper cone and examined in order to detect root-face alterations in the resected root end, such as marginal chipping. Finally, a zinc oxide EBA-reinforced cement (Super Seal; Ogna Pharmaceuticals, Milan, Italy) was used as root-end filling material (Figure 4). A fine diamond bur was used to remove the excess of filling material. The reflected tissues were reapproximated to

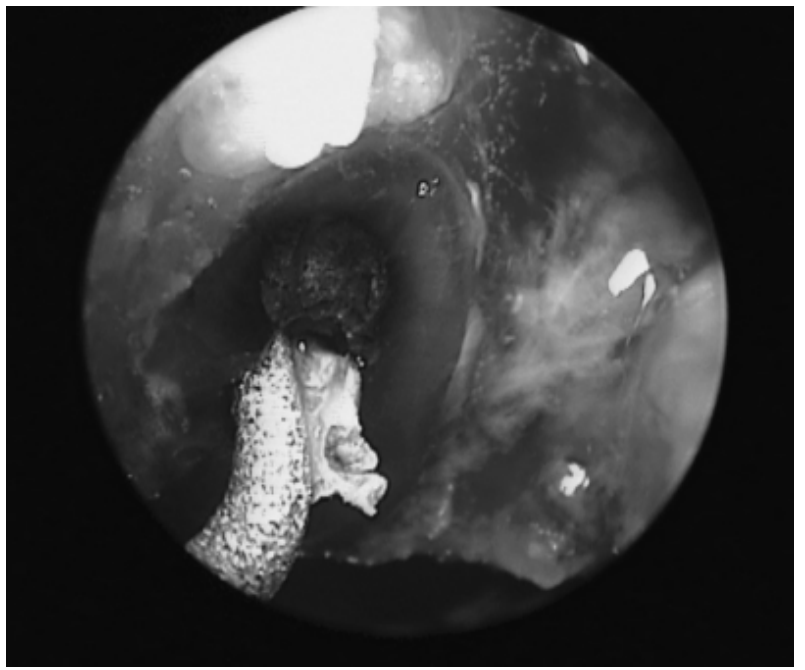

Figure 3 - Endoscopic view of the retro-tip during root-end cavity preparation.

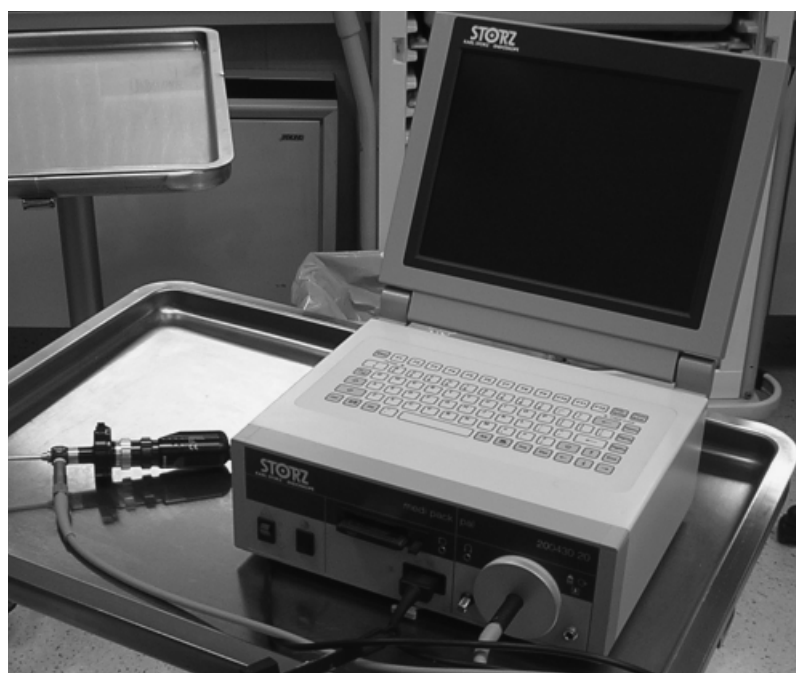

Figure 5 - Endoscopic components placed on a mobile rack.

their original position, compressed and stabilized, and sutured with non absorbable silk 5-0 (Ethicon Inc., Piscataway, NJ, USA).

\section{Endoscope in the surgical room}

Four main components, all placed on a mobile rack, make up the endoscope: (a) rod-lens system, (b) camera head, (c) camera control unit with a monitor and light source (Figure 5). The rack is fully maneuverable with its correct position being at the level of the patient's lower leg region on the opposite side of the surgeon. This allows the surgeon and the whole 


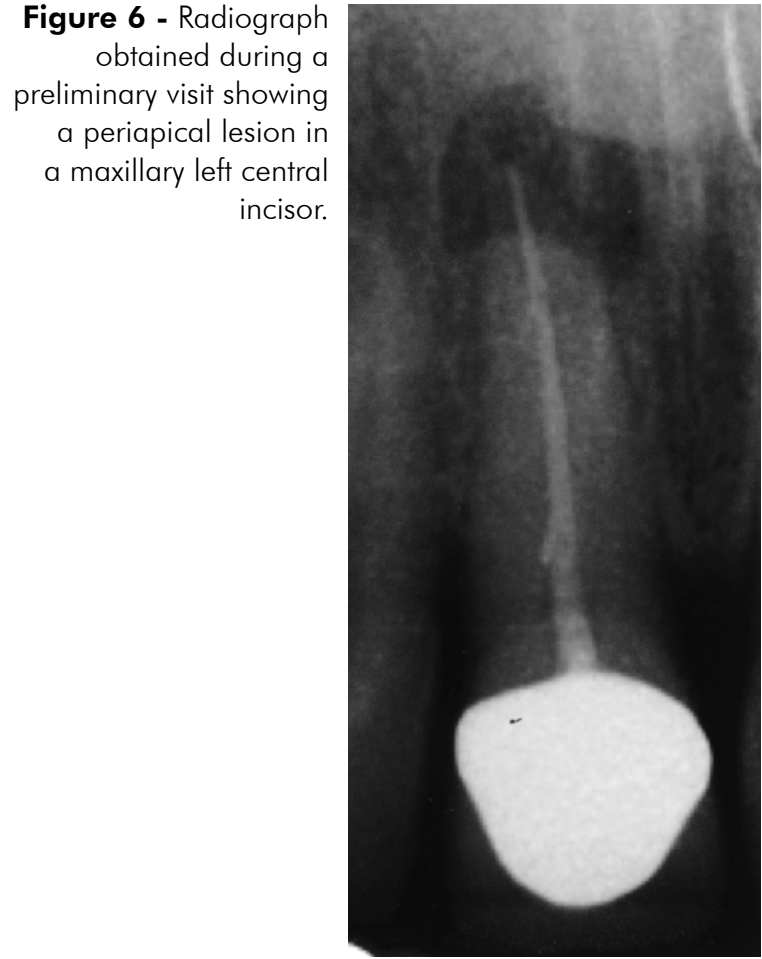

surgical team to view the surgery on the monitor. The surgeon holds the endoscope and the camera in one hand with a pen grasp. This way, the viewing angle and the position of the endoscope lens can be easily adjusted. For aseptic reasons, the video camera and the camera cable are packed in sterile covers and connected to the ocular of the endoscope.

\section{Criteria for healing assessment}

A radiograph of each tooth was taken during the preliminary visit (Figure 6). Further radiographs were taken at each clinical appointment $(3,6,12$ and 24 months). The paralleling technique was adopted to ensure reproducibility. The radiographs taken at 12 and 24 months were used to assess healing (Figure 7). The radiographic criteria used to assess the outcome were those established by Molven et al. ${ }^{13,14}(1987,1996)$.

At each clinical appointment, a clinical evaluation was recorded, following the guidelines of Gutmann, Harrison ${ }^{15}$ (1991).

According to modern concepts ${ }^{16}$ the success of endodontic treatment was evaluated not only in terms of radiographic and clinical healing but also in terms of functional retention of the tooth. A case

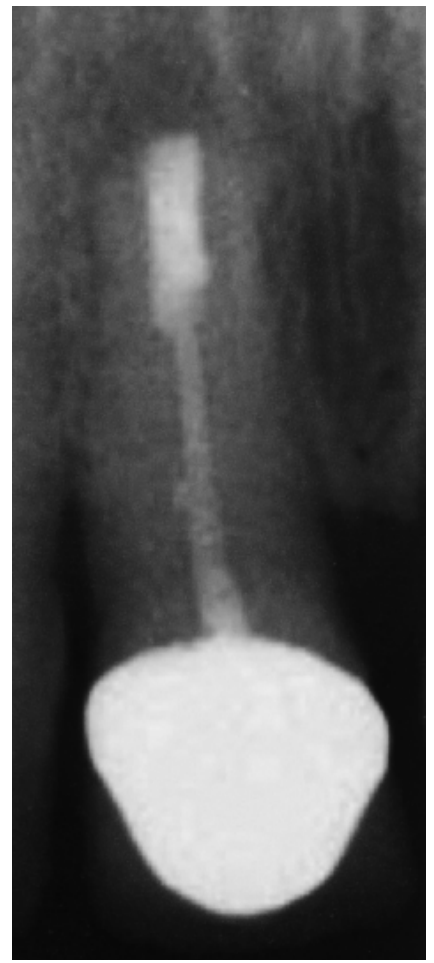

Figure 7 - Postoperative radiograph of the same case presented in figure 6, taken 24 months following surgery. Note the appearance of the healed tissue. was considered as functionally successful when clinical presentation was normal (asymptomatic tooth) regardless of the presence of radiolucency. All cases presenting with clinical symptoms were classified as not functional.

Fisher's exact test was used to statistically assess the difference between successful and unsuccessful cases for each of the variables considered. The tooth was considered as the unit of analysis. A probability value of $\mathrm{p}=0.05$ was considered as the level of significance.

\section{Results}

According to the selection criteria, 50 teeth in 34 patients ( 20 women, mean age 43 years and 14 men, mean age 37 years) were included in the study and treated with periradicular surgery.

One tooth was extracted during the surgical procedure before root-end resection because of vertical fracture, so the tooth (and the patient as well) was excluded from the study. Two patients accounting for 4 teeth did not come to the 1-year follow-up visit and were therefore excluded from the study. Another patient accounting for two teeth did not come to the 2-year follow-up visit. A final total of 43 teeth in 30 
patients were evaluated up to 2 years. Among them, 25 were in the maxilla (17 anterior, 6 premolar and 2 molar teeth) and 18 in the mandible (10 anterior, 4 premolar and 4 molar teeth). The maximum diameter of the periapical lesion ranged between 2.5 and $15 \mathrm{~mm}$.

After 1 year, 41 teeth out of the 45 evaluated were classified as successful (91.1\%), 2 as uncertain healing and 2 as failures. Forty-three cases were considered as functional $(95.5 \%)$. Of the 43 teeth evaluated at the 2-year follow-up, 39 (90.7 \%) healed successfully, 1 teeth had uncertain healing, and 3 were classified as failures. Forty cases were classified as functional (93\%) 2 years after surgery. It is noteworthy that of the two teeth classified as uncertain healing (and as functional) at the 1-year follow-up, one developed clinical symptoms during the second year and had to be extracted. The other one was still classified as uncertain healing (due to the persistence of radiolucency) at the 2-year follow-up. In agreement with other authors, ${ }^{14,17}$ the latter case was scheduled for a further follow-up 2 years later.

No statistically significant difference was found in treatment results between the 1- and 2-year evaluations neither in terms of healing $(\mathrm{p}=0.12)$ nor in terms of functionality $(\mathrm{p}=0.31)$. No difference related to tooth type $(\mathrm{p}=0.30)$ and tooth location (maxilla versus mandible, $\mathrm{p}=0.24$ ) was found at the 2-year follow-up.

\section{Discussion}

The present study included some patients whose outcomes have been previously reported ${ }^{8}$ but this one consists of an expanded cohort followed up for an extended time period.

In addition to evaluating the endoscope benefits in the surgical endodontic procedure, one of the aims of this study was to investigate a novel aspect of outcome assessment in endodontics, namely tooth functional retention, as proposed earlier by Friedman, $\operatorname{Mor}^{16}$ (2004).

Many clinical studies have been published on periradicular surgery using microsurgical retrotips. ${ }^{4,5,18-20}$ The introduction of ultrasonic retro-tips in periradicular surgery has brought many advantages in the procedure of root-end preparation. They enable the long axis of the tooth to be followed, while preserving the morphology of the canal. Apical cavities can be shaped easily, safely, and with greater precision as compared to using conventional handpieces. ${ }^{3,21} \mathrm{~A}$ well shaped root-end cavity, which is more centrally placed and smaller than that produced by microhandpieces and burs, can also reduce the risk of root perforation in deeply fluted roots and the inability to prepare to a sufficient depth. In addition, the utilization of ultrasonic retro-tips requires small bony crypts, and the cutting bevel obtained on the resected root end can be quite perpendicular to the canal's long axis. This fact might be beneficial because it decreases the number of exposed dentinal tubules at the resected root surface, minimizing apical leakage. ${ }^{3}$

Bader, Lejeune ${ }^{22}$ published in 1998 the first clinical study comparing ultrasonic and conventional root-end preparations. The success rates for ultrasonically treated teeth were significantly better than those for teeth treated with microburs. Other studies $^{19,23,24}$ showed similar differences between the 2 root-end preparations. A number of clinical studies have been published on periradicular surgery using microsurgical retro-tips, reporting success rates ranging between $82 \%$ and $96.8 \% .^{3,16}$ In general, the highest success rates have been reported when magnification devices were used. ${ }^{4}$

The use of high quality magnification devices in dentistry is becoming more and more common, with the aim of improving treatment quality. ${ }^{15}$ Many authors showed how these devices provide the visual access necessary to perform microsurgery with a greater degree of confidence and accuracy. ${ }^{4,5,25-27} \mathrm{~A}$ recent addition to the field of visualization devices is the fiberoptic endoscope.

Only two short-term clinical prospective studies concerning endoscopy in endodontic surgery were published. ${ }^{8,9}$ In the first published article the authors compared endodontic surgery with and without endoscopy. The success rates were not significantly different, $88.9 \%$ and $75.4 \%$, respectively. ${ }^{9}$ In the other study, the endoscope group presented a $94.9 \%$ success rate at the one-year follow-up versus $90.6 \%$ for the group in which surgical loupes were used. ${ }^{8}$ In the present study, the success rate was $91.1 \%$ at 
the one-year follow-up, and remained substantially unchanged one year later.

The main benefit considered in evaluating the outcome of surgical treatment is the probability of healing. However, the concept of tooth functional retention has been recently proposed as a further indicator of treatment success. In a literature review, Friedman, Mor ${ }^{16}$ (2004) showed that the chance of teeth with apical periodontitis to completely heal after apical surgery is highly variable among different studies if the success is evaluated according to conventional clinical and radiographic criteria (from $37 \%$ to $85 \%$ ), mainly due to great heterogeneity. In fact, many variables can affect the prognosis of the surgical treatment, such as: surgical procedure, materials, radiographic and clinical outcome assessment, the patient's systemic condition, type of tooth, quality of previous root canal treatment or re-treatment and of coronal restoration. Furthermore, one of the main reasons for the variability of reported outcomes is the different criteria for the evaluation of success and failure of the treatment that have been adopted. If functionality criteria are adopted, a much lower variability occurs (86 to $92 \%$ ), which

\section{References}

1. von Arx T, Walker WA. Microsurgical instruments for rootend cavity preparation following apicoectomy: A literature review. Endod Dent Traumatol. 2000 Apr;16(2):47-62.

2. Torabinejad M, Pitt Ford TR. Root-end filling materials: A review. Endod Dent Traumatol. 1996 Aug;12(4):161-78.

3. Kim S, Kratchman S. Modern endodontic surgery concepts and practice: A review. J Endod. 2006 Jul;32(7):601-23.

4. Rubinstein RA, Kim S. Short-term observation of the results of endodontic surgery with the use of a surgical operation microscope and Super-EBA as root-end filling material. J Endod. 1999 Jan;25(1):43-8.

5. Rubinstein RA, Kim S. Long-term follow-up of cases considered healed one year after apical microsurgery. J Endod. 2002 May;28(5):378-83.

6. Bahcall JK, Barss J. Orascopic visualization technique for conventional and surgical endodontics. Int Endod J. 2003 Jun;36(6):441-7.

7. Bahcall JK, Di Fiore PM, Poulakidas K. An endoscopic technique for endodontic surgery. J Endod. 1999 Feb;25(2):1325.

8. Taschieri S, Del Fabbro M, Testori T, Francetti L, Weinstein RL. Endodontic surgery using two different magnification seems to reflect more closely the generally observed tooth prognosis. ${ }^{16}$ In the present study, the difference in treatment outcome as evaluated according to the different criteria was found to be minimal. It can be hypothesized that adherence to a strict endodontic microsurgical procedure and an accurate case selection might be key factors in maximizing treatment success.

\section{Conclusion}

In the present study, the endoscope provided excellent visualization of the surgical field during rootend management and can be an aid for endodontic surgical procedure in terms of both healing and functionality.

Further clinical trials with standardized protocols and a high level of evidence are needed to confirm the results of the present study.

\section{Acknowledgements}

The Authors wish to thank Mr. Tom Lacey for his support in checking the English language of the manuscript.

devices: Preliminary results of a randomized controlled study. J Oral Maxillofac Surg. 2006 Feb;64(2):235-42.

9. von Arx T, Frei C, Bornstein M. Periradicular surgery with and without endoscopy: A prospective clinical comparative study. Schweiz Monatsschr Zahnmed. 2003;113(8):860-5.

10. von Arx T, Hunenbart S, Buser D. Endoscope- and video-assisted endodontic surgery. Quintessence Int. 2002 Apr;33(4):255-9.

11. von Arx T, Montagne D, Zwinggi C, Lussi A. Diagnostic accuracy of endoscopy in periradicular surgery - A comparison with scanning electron microscopy. Int Endod J. 2003 Oct;36(10):691-9.

12. Taschieri S, Del Fabbro M, Testori T, Francetti L, Weinstein RL. Combining surgical microscope and endoscope for achieving excellent aesthetic outcome in soft tissue healing and maximizing the success of periradicular surgery. Pract Periodont Esthet Dent. 2006;18:193-8.

13. Molven O, Halse A, Grung B. Observer strategy and the radiographic classification of healing after endodontic surgery. Int J Oral Maxillofac Surg. 1987 Aug;16(4):432-9. 
14. Molven O, Halse A, Grung B. Incomplete healing (scar tissue) after periapical surgery. Radiographic findings 8-12 years after treatment. J Endod. 1996 May;22(5):264-8.

15. Gutmann JL, Harrison JW. Surgical Endodontics. Boston: Blackwell Scientific Publications; 1991.

16. Friedman S, Mor C. The success of endodontic therapy - healing and functionality. J Calif Dent Assoc. 2004 Jun;32(6):493503.

17. Rud J, Andreasen JO, Möller Jensen JE. A follow-up study of 1000 cases treated by endodontic surgery. Int J Oral Surg. 1972;1(4):215-28.

18. Chong BS, Pitt Ford TR, Hudson MB. A prospective clinical study of mineral trioxide aggregate and IRM when used as root-end filling materials in endodontic surgery. Int Endod J. 2003 Aug;36(8):520-6.

19. Testori T, Capelli M, Milani S, Weinstein RL. Success and failure in periradicular surgery. A longitudinal retrospective analysis. Oral Surg Oral Med Oral Pathol Oral Radiol Endod. 1999 Apr;87(4):493-8.
20. Zuolo ML, Ferreira MOF, Gutmann JL. Prognosis in periradicular surgery: A clinical prospective study. Int Endod J. 2000 Mar;33(2):91-8.

21. Plotino G, Pameijer CH, Grande NM, Somma F. Ultrasonics in endodontics: A review of the literature. J Endod. 2007 Feb;33(2):81-95.

22. Bader G, Lejeune S. Prospective study of 2 retrograde endodontic apical preparations with and without the use of $\mathrm{CO}_{2}$ laser. Endod Dent Traumatol. 1998 Apr;14(2):75-8.

23. Engel TK, Steiman HR. Preliminary investigation of ultrasonic root-end preparation. J Endod. 1995 Sep;21(9):443-5.

24. Mehlhaff DS, Marshall JG, Baumgartner JC. Comparison of ultrasonic and high-speed-bur root-end preparations using bilaterally matched teeth. J Endod. 1997 Jul;23(7):448-52.

25. Cohen S, Burns RC. Pathways of the Pulp. $8^{\text {th }}$ ed. St. Louis: Mosby; 2002.

26. Friedman M, Mora AF, Schmidt R. Microscope-assisted precision dentistry. Compend Contin Educ Dent. 1999 Aug;20(8):723-8.

27. Kim S. Modern endodontic practice. Instruments and techniques. Dent Clin North Am. 2004 Jan;48(1):1-9. 